\section{Die magnetische Widerstandsänderung in InAs}

Von H. WeIss

Forschungslaboratorium der Siemens-Schuckertwerke, Erlangen

(Z. Naturforschg. 12 a, 80 [1957] ; eingegangen am 1. Dezember 1956)

Eine hohe Elektronenbeweglichkeit ist im allgemeinen mit einer großen magnetischen Widerstandsänderung verbunden. So ist der spezifische Widerstand eines stabförmigen Präparates aus $\mathrm{InSb}$ in einem Magnetfeld von $10000 \mathrm{Gau} ß$ bei Zimmertemperatur etwa doppelt so groß wie ohne Magnetfeld. Im Gegensatz dazu zeigt ein einkristallines Stäbchen aus $\operatorname{InAs}^{1}$ einen ungewöhnlich niedrigen Effekt. Um diesen zu erhalten, muß man den Einfluß der geometrischen Form des Versuchskörpers berücksichtigen. Dazu wurden folgende Messungen durchgeführt:

An ein einkristallines Stäbchen (Querschnitt $2 \times 2 \mathrm{~mm}^{2}$, Länge $25 \mathrm{~mm}, \mathrm{H}_{\mathrm{ALL}}-$ Koeffizient $R_{\mathrm{H}}=-115 \mathrm{~cm}^{3} /$ Asec, Elektronenbeweglichkeit

$$
\mu=\frac{8}{3 \pi} R \sigma=16200 \mathrm{~cm}^{2} / \mathrm{Vsec},
$$

Längsachse parallel zur [111]-Richtung) wurden an beiden Enden Stromzuführungen gelötet. Über die ganze Länge des Präparates, zwischen den punktförmigen Sonden A und B in Abb. 1, betrug die Widerstandsänderung in einem Magnetfeld von $10000 \mathrm{Gau} ß$ $11 \%$ (Kurve $\mathrm{AB}$ ). Wurde die Widerstandsänderung mit zwei Sonden C und D gemessen, die sich innerhalb der Enden befanden, so war sie um so kleiner, je größer der Abstand der Sonden von den Enden war. Betrug er jeweils $4,5 \mathrm{~mm}$, so änderte sich der Widerstand bei $10000 \mathrm{Gauß}$ nur um 2,4\% (Kurve CD). Rückten die Sonden noch näher zusammen, so blieb dieser Wert erhalten. Daraus folgt, daß die "physikalische“ magnetische Widerstandsänderung in InAs durch die Kurve $\mathrm{CD}$ wiedergegeben wird, wobei

$$
\Delta \varrho / \varrho=\text { const } \cdot B^{1,65} .
$$

Bei dieser Messung mußte eine Schwierigkeit berücksichtigt werden, die durch den großen $\mathrm{H}_{\mathrm{ALL}}$-Winkel bedingt ist: ist der HALL-Koeffizient nicht über die Länge der Probe konstant, sondern besitzt er einen Gradienten, so kann es vorkommen, daß die gemessene magnetische Widerstandsänderung beim Umpolen des Magnetfeldes wesentlich andere, unter Umständen negative Werte zeigt. Es konnte daher nur ein homogener Einkristall zu diesen Messungen verwendet werden.

Der Unterschied zwischen den beiden Kurven AB und CD rührt daher, daß in dem Gebiet vor den Stromelektroden die Hall-Spannung verschwindet. Dann sind dort die Strombahnen um den HaLL-Winkel gedreht

1 R. Gremmelmaier, Z. Naturforschg. 11 a, 511 [1956].

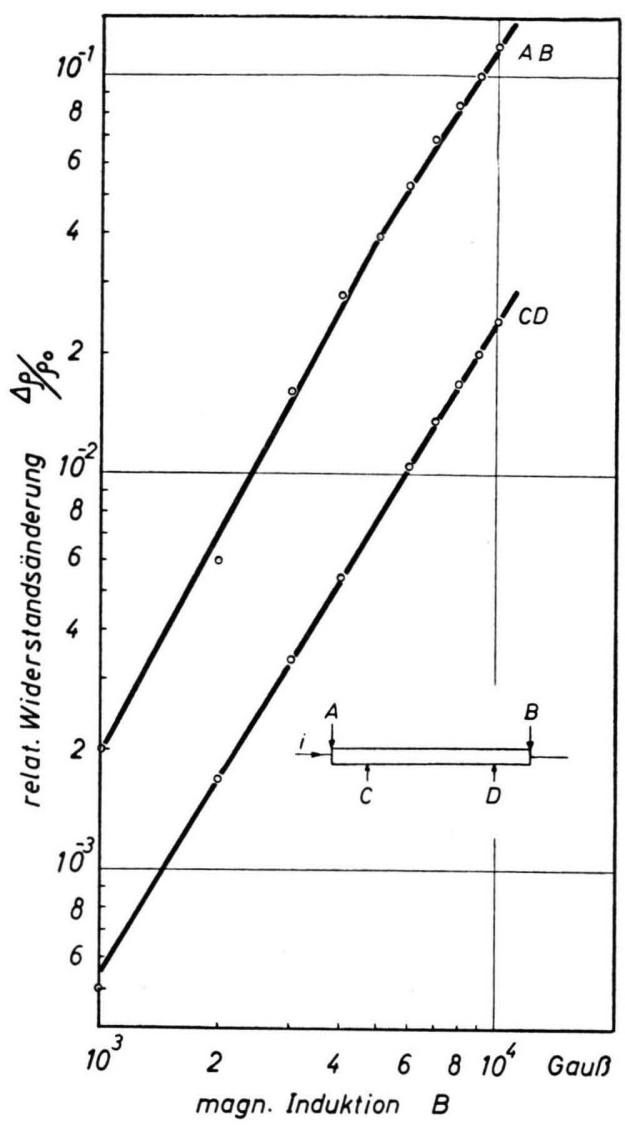

Abb. 1.

und man erhält eine „geometrische“ Widerstandsänderung, wie sie WeIss und $\mathrm{W}_{\text {ELKER }}{ }^{2}$ an Rechteckplatten beobachtet haben. Die Verfasser haben gezeigt, daß der Einfluß der Geometrie auf die magnetische Widerstandsänderung um so größer ist, je größer das Verhältnis von Elektronenbeweglichkeit zu Widerstandsänderung an einer stabförmigen Probe ist. Dieses Verhältnis ist bei InAs so groß, daß noch bei einem Stäbchen mit Länge : Breite $=25: 2$ die magnetische Widerstandsänderung durch die Geometrie mehr als vervierfacht wird. Eine Corbinoscheibe aus InAs zeigt bei $10000 \mathrm{Gau} ß$ den 5,5-fachen Widerstand. Für die kleine „physikalische“ Widerstandsänderung von nur $2,4 \%$ bei $10000 \mathrm{Gauß,} \mathrm{die} \mathrm{für} \mathrm{den} \mathrm{unendlich} \mathrm{langen}$ Stab gilt, gibt es noch keine Erklärung.

Für die Überlassung der zur Messung erlorć rlichen Einkristalle danke ich Herrn Dr. Gremmelmaier.

${ }^{2}$ H. Weiss u. H. Welker, Z. Phys. 138, 322 [1954]. 